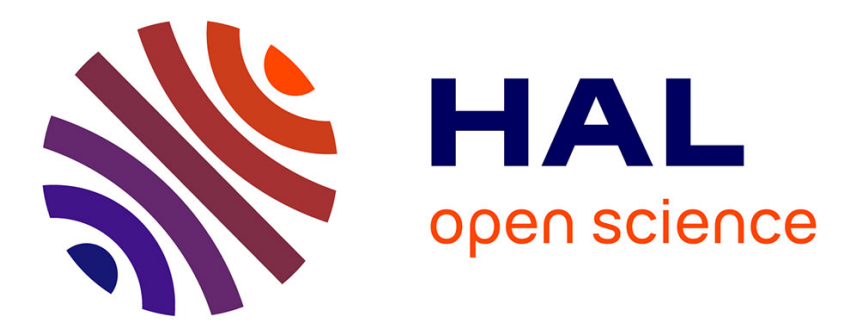

\title{
Cartographie et processus d'Intelligence Economique: L'analogie du plateau de jeu comme aide à la décision stratégique \\ Stéphane Goria
}

\section{- To cite this version:}

Stéphane Goria. Cartographie et processus d'Intelligence Economique : L'analogie du plateau de jeu comme aide à la décision stratégique. Les Cahiers du numérique, 2009, Les Cahiers du Numérique, 5 (4), pp.111-137. 10.3166/lcn.5.4.111-137 . halshs-00461699

\section{HAL Id: halshs-00461699 \\ https://shs.hal.science/halshs-00461699}

Submitted on 3 Nov 2016

HAL is a multi-disciplinary open access archive for the deposit and dissemination of scientific research documents, whether they are published or not. The documents may come from teaching and research institutions in France or abroad, or from public or private research centers.
L'archive ouverte pluridisciplinaire HAL, est destinée au dépôt et à la diffusion de documents scientifiques de niveau recherche, publiés ou non, émanant des établissements d'enseignement et de recherche français ou étrangers, des laboratoires publics ou privés.

\section{(c)(1)}

Distributed under a Creative Commons Attribution| 4.0 International License 


\title{
CARTOGRAPHIE ET PROCESSUS D'INTELLIGENCE ÉCONOMIQUE
}

\author{
L'analogie du plateau de jeu comme aide à la décision \\ stratégique
}

STEPHANE GORIA

LORIA - Université de Lorraine (stephane.goria@loria.fr)

\section{Introduction}

L'intelligence économique s'intéresse avant tout à l'information à travers sa collecte, sa transformation, sa diffusion ainsi qu'à son emploi par et pour les décideurs. À travers ces différents centres d'intérêt, elle vise notamment à fournir une information à valeur ajoutée aux décideurs. En ce sens, tout moyen d'aide à l'explicitation de problème et de mise en valeur de l'information peut être considéré comme un atout pour l'intelligence économique. Nos recherches s'intéressent à ce type de moyens et plus particulièrement à la mise en valeur de l'information à partir de représentations visuelles. Pour ce faire, nous présentons ici l'apport d'une forme particulière de cartographie pour aider à la mise en valeur de l'information. À ce stade, nous ne faisons pas la distinction entre information et connaissance au niveau cartographique pourvu qu'il s'agisse d'un moyen de mise en forme qui focalise notre attention, stimule nos capacités créatives et aide à la mémorisation. En ce sens, comme le signale (Lecocq, 2005) l'expression «cartographie des connaissances » peut être considérée sous un spectre très large et être à la fois synonyme : de représentation de processus permettant de recenser et de catégoriser les connaissances d'une organisation, d'audit de connaissances, de visualisation d'ontologies, de cartes cognitives de système de localisation d'experts, de mode de représentation graphique permettant la localisation de certaines connaissances ainsi que les liens les unissant. Dans l'optique qui nous intéresse, nous considérons les sens ci-dessus accordés à la cartographie comme une liste d'objectifs à laquelle elle peut contribuer. Toutefois, nous centrons actuellement nos travaux surtout sur l'apport des représentations graphiques pour aider à identifier des formes spécifiques liant différentes informations. Dès lors, notre problématique peut s'exprimer ainsi : quel moyen peut-on mettre en œuvre pour aider à l'identification de stratégies mises en place ou mises en œuvre, au repérage de signaux d'une évolution en cours, à la compréhension d'un problème décisionnel ${ }^{1}$ et de son contexte ? De la sorte, nous partons de l'hypothèse principale, que la représentation graphique d'éléments assimilables à des territoires peut aider à résoudre ce type de problématique. Le corollaire qui en découle considère que les représentations graphiques facilitent le raisonnement territorial. De cette manière, il nous faut étudier ce qu'est le territoire et ce qu'il peut permettre comme analogies pour aider à résoudre des problèmes. La carte est alors considérée comme une passerelle entre une représentation territoriale de l'ordre de la pensée et une représentation graphique d'informations. Réciproquement, la cartographie d'informations permet d'élaborer des stratégies territoriales ou de mieux comprendre leur mise en œuvre. Dans cet ordre d'idées, nous fondons nos travaux sur une hypothèse sous-jacente à la principale qui suppose que l'analogie du jeu de stratégie sur plateau contribue à amélioration de l'expression de problèmes ainsi qu'à la compréhension de certains phénomènes.

Il existe déjà de nombreux outils qui ont été employés pour cartographier des territoires qu'ils soient réels, virtuels ou de pures abstractions (Goria, 2007 ; Humbert et al., 2008). Cependant, nous constatons que le potentiel de représentations territoriales fourni par les jeux de stratégie sur plateau est quasi inexistant en intelligence économique. Or, ces jeux sont très nombreux, pour certains relativement connus et offrent un réservoir de solutions intéressantes pour aider au processus d'intelligence économique, d'autant que des travaux en relation avec le Management

1. Pour le moment, nous nous concentrons surtout sur des problèmes décisionnels pouvant être déclinés en problème(s) de recherche d'information, de veille (stratégique, concurrentielle, etc.), d'influence, d'attractivité territoriale ou d'innovation. 
Stratégique des organisations se sont déjà intéressés à ce potentiel (Saucin, 2004 ; Slywotzky, 1999 ; Fauvet et al., 1997) ou (Thiriez, 1995).

Les jeux que nous étudions emploient un plateau sur lequel les deux dimensions du plan sont exploitées et où il est question de contrôle, d'utilisation et de conquête de territoires. Photographiée à un instant $\mathrm{t}$, une partie de l'un de ces jeux permet une analogie assez simple pour élaborer des représentations territoriales en deux dimensions. Selon l'analogie employée et l'exploitation de certains éléments de base des règles et des stratégies de ces jeux, nous défendons l'idée que les cartographies qu'ils proposent créent une valeur ajoutée pour le processus d'intelligence économique. Par exemple, la mise en forme de signaux informationnels sous la forme d'indices de développement territoriaux liés au jeu de référence, selon nous, apporte des solutions originales pour créer une valeur ajoutée visuelle à de l'information déjà identifiée par un veilleur. Pour argumenter cette idée, nous abordons dans ce papier, d'abord les rapports qui peuvent exister entre la cartographie et l'intelligence économique ainsi que les apports de l'un à l'autre de ces domaines. Ensuite, nous étudions le concept de territoire pour le mettre en perspective avec ses mises en forme cartographiques et leurs contributions à la résolution de problèmes. Puis, nous présentons l'analogie du jeu de stratégie sur plateau à partir de son positionnement vis-à-vis du concept de territoire et la résolution de problèmes, mais surtout en présentant plusieurs jeux de ce type pour mettre en évidence leur potentiel analogique. Dans le prolongement de cette idée, nous abordons par la suite la transition entre les jeux de stratégie «classiques » et les wargames à partir d'une petite présentation de ces derniers. Enfin, nous proposons un tableau récapitulatif des caractéristiques des différents jeux présentés dans la perspective de la mise en forme d'une aide au choix d'une cartographie fondée sur l'analogie du jeu de stratégie sur plateau.

\section{Intelligence économique et cartographie d'informations}

Depuis que l'expression intelligence économique a été employée et associée à une définition via le rapport dit Martre (Martre et al., 1994), ce terme n'a cessé d'évoluer dans sa forme, ses définitions et ses multiples mises en œuvre. Toutefois, quel que soit le point de vue choisi pour y référer, elle a pour principal objectif d'aider les décideurs dans leur relation avec l'environnement informationnel. Parmi, les choix de définition de l'intelligence économique qui ont pu être réalisés, deux catégories sont un peu plus couramment employées que les autres (Goria, 2007). La première considère l'intelligence économique comme un processus et la seconde comme un ensemble de fonctions. C'est à partir de ses deux catégories de définitions que nous nous appuyons pour justifier l'intérêt de la cartographie d'informations pour l'intelligence économique.

\section{Rapport entre la cartographie et le processus d'intelligence économique}

Il existe différentes propositions de l'intelligence économique considérée sous forme de processus. Certaines l'assimilent au cycle du renseignement, d'autres vont plus loin comme (Galland et al., 2008) ou (Guilhon, 2004). Parmi celles-ci, nous nous fondons sur l'expression du processus d'intelligence économique donnée par David et al., (2003) :

- définition du problème décisionnel ;

- transformation du problème décisionnel en problème de recherche d'information ;

- identification de sources d'informations pertinentes ;

- recherche des informations pertinentes ;

- traitement, analyse et diffusion des informations ;

- interprétation des informations collectées et transformées par le décideur ;

- décision.

Selon un tel processus et notre hypothèse de recherche, la cartographie d'informations est en mesure de créer une valeur ajoutée pour l'intelligence économique à aux moins deux niveaux. D'abord, lors de la transformation du problème décisionnel en problème de recherche d'informations. Il s'agit de la phase d'explicitation de son problème par le décideur au veilleur par exemple. Une représentation graphique des informations peut alors jouer différents rôles : aider le décideur à mieux comprendre son problème en mettant littéralement «à plat » son 
raisonnement, fournir un support média pour discuter, comprendre et pointer certains éléments à traiter ou à ne pas traiter, présenter les informations à rechercher pour mieux les identifier et les hiérarchiser. Ensuite, lors de la présentation des informations au décideur, la cartographie graphique permet de compléter un document textuel en proposant : une hiérarchie visuelle des éléments classés, une structure qui donne naissance à une compréhension schématique d'un ensemble d'informations, un plan de réflexion différent ou une redondance visuelle de certaines informations pour aider à mieux les mémoriser ou reconnaître (la carte géographique en est l'exemple le plus connu).

\section{Rapport des fonctions d'intelligence économique à la cartographie}

Lorsque l'on considère l'intelligence économique selon un spectre général, on lui associe en général trois fonctions (AFDIE, 2005) : 1) la Veille, 2) la Gestion du risque informationnel, 3) l'Influence et le lobbying. Dans le cas de son application à un territoire, on peut également lui ajouter la fonction : Création et gestion de réseaux humains et de clusters d'entreprises (Clerc, 2004). Certaines autres fonctions peuvent aussi être comprises sous l'appellation intelligence économique ou la compléter directement. Parmi celles-ci, les plus courantes sont la Gestion des connaissances et l'Assistance à l'innovation (SCIE, 2009 ou Goria et al., 2009). Ceci nous ramène à un spectre d'intelligence économique étendu à six fonctions. Nous allons considérer chacune de ces fonctions dans ses rapports à la cartographie d'informations ou de connaissances pour mieux mettre en évidence la contribution élargie d'une cartographie à valeur ajoutée pour l'intelligence économique.

\section{Cartographie et veille}

La veille se décline en diverses catégories: technologique, marché, sectorielle, concurrentielle, stratégique, territoriale... En tant que veille technologique, elle tend à suivre les changements technologiques et à anticiper les ruptures technologiques en identifiant certains signaux dits «faibles». Si l'on dispose alors de moyens pour cerner un champ technologique et envisager ses possibles évolutions, on ne peut que contribuer à ce type de veille. Du point de vue des veilles marché, sectorielle et concurrentielle, il s'agit de suivre l'évolution de son secteur d'activité, de ses concurrents, de ses marchés et de son propre positionnement vis-à-vis de ces derniers. Tout moyen pour représenter ce positionnement ne peut dès lors que rendre service à ce type de veille. Dans son optique stratégique, la veille vise, notamment, à surveiller, à anticiper ainsi qu'à découvrir les stratégies des organisations qu'elle cible. De ce fait, si une méthode, même analogique, permet de mettre en évidence certaines stratégies des organisations, ce moyen ne peut qu'aider à la veille stratégique. Dans un registre similaire, les veilles prospective et territoriale visent à envisager de nouveaux champs d'action ainsi qu'à surveiller un territoire et ses évolutions. Bien sûr, il existe encore d'autres veilles (produit, image, économique, sociale, juridique, fournisseurs...), mais selon les cas, nous pensons que, au moins occasionnellement, la cartographie d'un environnement ou l'analogie territoriale peuvent aussi y être employées pour mieux mettre en évidence certaines informations-clés.

\section{Cartographie et gestion du risque informationnel}

La gestion du risque informationnel peut se décliner sous différentes formes : Audit de sûreté, Sécurité informationnelle, Sensibilisation et aide à la gestion de la propriété intellectuelle, Gestion et communication de crise (Lancini et al., 2007). Selon ces différentes formes, la Gestion du risque informationnel peut être traitée avec l'aide d'un outil de cartographie d'un environnement perçu en tant que territoire. Ainsi, lors d'un audit de sécurité et de ses mises à jour, il peut être intéressant de cartographier l'organisation et de positionner certains des risques qu'elle encoure. Concernant, la Gestion et la communication de crise, les war-rooms qui sont des espaces d'aide à la décision dédiés en partie à ces cas de figure sont habituellement des pièces où la cartographie d'informations joue un rôle essentiel puisqu'elle tapisse certains des murs et tables de ces environnements décisionnels (Goria, 2008). 


\section{Cartographie et influence et lobbying}

La représentation des interactions entre acteurs influents sur un sujet donné, que ce soit directement du point de vue humain ou via des médias tels les blogs et les forums du web, prend souvent la forme d'une cartographie (Joachim et al., 2006). Il s'agit d'identifier des nœuds et des leviers d'influence pour mieux faire circuler et valoir les informations que l'on souhaite communiquer. Réciproquement, pour toute politique d'influence, il est nécessaire de comprendre, dans la perspective choisie, quelles sont les stratégies d'influence et de lobbying déjà mises en œuvre par la concurrence ainsi que celles sur lesquelles s'appuient déjà nos partenaires (Romma et al., 2005). De plus, il est toujours intéressant pour toute stratégie d'influence ou de lobbying, d'identifier des carrefours informationnels sensibles ou critiques ainsi que des agents isolés qui pourraient s'avérer utiles pour la stratégie choisie. Or, la cartographie d'informations fournit quelques moyens graphiques pour aider à répondre à ce type de besoin.

\section{Cartographie et création et gestion des réseaux humains et de clusters d'entreprises}

Dans le cadre de nombreux dispositifs d'intelligence économique territoriale qui ont été mis en place, la Création ou la gestion d'un cluster d'entreprises est l'un des axes majeurs du dispositif (Pautrat et al., 2009). Dès lors, que l'on soit dans l'optique du développement d'un cluster, d'un système productif local ou de tout autre pôle de compétitivité, il est essentiel de se positionner au moins vis-à-vis de l'environnement géographique, concurrentiel et technologique. Similairement à la veille stratégique, tout moyen de représentation territoriale visant à cartographier les champs d'implication de l'organisation ne peut que lui être utile. Concernant, la représentation géographique du territoire, le nombre de systèmes d'information géographique qui sont employés par ces catégories de dispositifs rend bien compte de l'utilité pour ses organismes de disposer d'outils de cartographie de leur environnement (Delvoye et al., 2005). Enfin, du point de vue de la gestion des réseaux humains, de la même manière qu'une cartographie d'informations peut mettre en évidence des réseaux d'influence, elle peut contribuer à la mise en évidence de réseaux humains en général.

\section{Cartographie et gestion des connaissances}

Selon les auteurs, la gestion des connaissances peut inclure différentes sous-fonctions (Goria, 2007): capitalisation des connaissances, cartographie des connaissances, acquisition des connaissances, partage de savoirs et création de connaissances. Parmi celles-ci, la cartographie des connaissances justifie de par son appellation son rattachement aux moyens de cartographie. Concernant l'acquisition et la capitalisation de connaissances, parmi les moyens souvent cités pour participer à l'élicitation de connaissances, nous retrouvons, par exemple, les outils comme les schémas heuristiques ou de mise en forme graphique du résultat d'un storytelling (Eppler et al., 2006). Du point de vue des fonctions création et partage de connaissances, ces dernières renvoient à l'utilité de la cartographie lorsque cette dernière est employée pour des projets collaboratifs où les cartes servent de support de médiation entre individus ou lorsque la cartographie est employée pour identifier des réseaux de personnes ou stimuler leurs capacités créatives de la même manière que dans le cadre d'une assistance à l'innovation (Buisine et al., 2009).

\section{Cartographie et assistance à l'innovation}

Dans le cadre de l'assistance à l'innovation et particulièrement dans la phase de stimulation de la créativité, les cartographies d'informations jouent un rôle important. Déjà les cartes cognitives, heuristiques ou conceptuelles sont employées pour soutenir des démarches de brainstorming. D'autres moyens de cartographie graphiques sont encore cités, ils sont utilisés dans le cadre de la mise en ouvre de stratégies d'innovation ou de projets collaboratifs pour innover (Anthony et al., 2006), mais aussi pour préparer le design d'un produit en permettant une représentation des attentes du consommateur ou de son point de vue sur une marque ou un produit (Bouzdine-Chameeva et al., 2007). En outre, des outils plus classiques comme les diagrammes causes-effets ou les matrices d'analyse morphologique peuvent être employés pour aider à identifier des pistes d'innovation, des besoins à combler, des problèmes à résoudre.

\section{Cartographie et territoire}

Comme nous l'avons précisé en première partie, notre hypothèse de recherche considère avant tout l'apport de la cartographie d'informations dans l'optique où elle est en mesure de 
fournir une aide au raisonnement territorial. De fait, il nous faut établir le lien qui existe entre les cartographies et le territoire.

\section{Le concept de territoire}

Les définitions associées au terme «territoire» ne manquent pas. Le grand dictionnaire terminologique ${ }^{2}$ et le Trésor de la Langue Française informatisé ${ }^{3}$ en proposent plusieurs selon les domaines d'application. On peut les résumer ainsi, lorsque l'on fait référence à un territoire, il peut s'agir d' :

- un lieu, une étendue de terre, un espace où sont circonscrits une activité, un phénomène ;

- un espace borné par des frontières, soumis à une autorité qui lui est propre, considéré en droit comme un élément constitutif de l'Etat et comme limite de compétence de ses gouvernants ;

- une assise géographique ou, plus particulièrement, un espace délimité où vit une communauté humaine, un individu, un couple ou une communauté animale ;

- une zone défendue par un occupant pour empêcher ses congénères ou d'autres animaux de s'y installer (cette zone est en général marquée, le plus souvent sous forme olfactive);

- un espace qu'une personne considère comme sien, où elle se sent à l'aise.

Champolion, (2006) a proposé une revue des définitions du territoire en rapport avec les sciences de l'éducation, les sciences de l'information et de la communication, la géographie et la sociologie. Il en a retiré cinq éléments clés qui caractérisent un territoire : un ensemble de ressources, une construction, un certain regard vers le futur, des effets spécifiques, des tensions entre le niveau local et global qui sont produits par ses membres. (Dumas et al., 2007) ont ajouté à ces éléments le fait qu'un territoire est avant tout une construction de l'esprit qui ne peut se définir qu'en rendant compte du point de vue de la personne qui a posé la question à laquelle la représentation territoriale répond. Dans le domaine de la publicité, Lendrevie et al., (2004) définissent un territoire de marque comme l'espace de valeurs, d'attentes où la marque est légitime aux yeux de la clientèle actuelle ou potentielle. Il détermine le champ de l'extension de marque, c'est-à-dire la possibilité d'utiliser la même marque pour des produits différents. .

En conclusion, dans le cadre de nos travaux, un territoire peut être considéré comme une réponse à une question qui se représente sous la forme d'un espace borné de manière plus ou moins nette et qui rend compte de phénomènes qui ont lieu en son sein ou de confrontations avec d'autres territoires. C'est à partir de ces fondements que nous raisonnons sur le territoire pour élaborer ses représentations cartographiques.

\section{Cartographie de territoire et résolution de problème}

Il est très difficile de parler de territoire sans en avoir une représentation. Dans une perspective visuelle, c'est la Cartographie qui s'est chargée de réaliser le transfert entre une représentation mentale, le plus souvent, du monde géographique et ses représentations visuelles pour mieux gérer le territoire et mieux communiquer sur et à propos de ce dernier. L'utilisation de représentations spatiales du type cartographique possède alors l'avantage de servir de support de médiation entre individus sur la base d'un raisonnement spatial (Vinck, 1999 ; Lardon et al., 2001). De la sorte, la représentation partagée d'un même territoire associée à une présentation des choix stratégiques potentiels ou réalisés facilite la résolution de problèmes (Lardon et al., 2008). Zhang, (2000) considère que pour raisonner sur un espace donné, deux sortes de représentations cognitives sont mises en œuvre : des représentations internes et des

2. http://www.olf.gouv.qc.ca/ressources/gdt.html

3. http://atilf.atilf.fr/tlf.htm 
représentations externes. Les représentations internes sont supposées être des structures mentales de connaissances qui aident les personnes à raisonner et à interagir avec leur environnement. Elles incluent des représentations de plusieurs types (propositionnelles, procédurales, distribuées, etc.) dont des représentations dites analogiques qui nous intéressent ici, car elles rendent compte des visions que nous avons du monde. Ce sont des connaissances organisées de façon à traduire sous la forme d'images mentales, en quelque sorte, la réalité qui nous entoure telle que la représentation mentale d'une carte de France qui n'est ni la carte elle-même, ni la France, mais dont chacun est capable de se faire une représentation. Les représentations externes rendent compte essentiellement de formes et de positions de symboles ainsi que de relations spatiales. En outre, il n'est pas nécessaire pour l'utilisateur de la cartographie de se construire un modèle mental du territoire, car la représentation visuelle permet à la solution du problème d'être directement perçue et utilisée sans être formulée explicitement. De même, Casakin et al., (1999) signalent d'après leurs expériences que l'analogie visuelle contribue autant chez le public expert que chez les novices à augmenter significativement les capacités de résolution de problèmes.

Toutefois, l'analogie visuelle ne doit pas piéger l'utilisateur. Dans la mesure du possible, les représentations proposées doivent être en rapport avec les représentations internes de l'utilisateur. Dans le cas contraire, l'apprentissage de l'utilisation du modèle doit être approfondi. (Gick et al., 1983 ; Nogry et al., 2007). En effet, malgré de nombreux avantages, les représentations cartographiques sont à manipuler avec précaution, car comme le signale Lempert (2002), les cartes sont aussi celles d'un poker menteur. Miroir de représentations qui sousentendent une rivalité de pouvoir dont l'objet est, et demeure, le territoire. Ce paramètre pris en compte, la carte possède comme propriétés essentielles de rendre compte de l'ordre géographique et de ce fait elle fournit la composante la plus pratiquement divisible, celle dans laquelle l'homme est capable d'identifier le plus grand nombre de catégories sans devoir faire intervenir des appareils ou des définitions complexes. (Bertin, 1968).

\section{Du territoire au plateau de jeu de stratégie}

Hunt et Menon, (1995) sur la base d'un travail de Arndt, (1985) ont retenu cinq métaphores qui rendent compte en général du concept de territoire: la guerre, l'organisme, le jeu, une étendue limitée de terre et le mariage. Les jeux de stratégie sur plateau ont la particularité de permettre l'emploi de trois de ces métaphores. En effet, le plateau de jeu est bien une étendue limitée et de nombreux jeux de stratégie correspondent par analogie à un affrontement militaire où de fait, le plateau représente souvent une étendue de terre. En nous intéressant aux jeux de stratégie sur plateau, nous disposons donc des avantages offerts par ces trois métaphores. Cette analogie du plateau de jeu de stratégie au territoire n'est pas nouvelle, Borzakian, (2009) et Bizet et al., (1997) y ont déjà travaillé. Selon ces auteurs, l'appellation «jeux de plateau » (de l'anglais boardgame) se distinguent des jeux de société « classiques » par le fait que ce sont avant tout des jeux de simulation qui font appel à des logiques spatiales, de concentration et, d'interactions entre lieux. Ils ont, notamment, remarqué qu'en plus du caractère esthétique du plateau qui est toujours très soigné, la qualité de la cartographie procure un plus grand plaisir de jeu en dehors de l'intérêt du jeu lui-même (Bizet et al., 1997). De fait, le plateau donne au joueur l'envie d'être exploité. De plus, ces jeux exigent des joueurs qu'ils orientent leurs décisions en fonctions des données telles que les distances et leur coût, ou encore, la position stratégique des territoires qu'ils contrôlent où veulent conquérir. (Borzakian, 2009). De même, le jeu a déjà été utilisé pour générer des interactions lors de réflexions sur le territoire pour identifier par exemple des actions à mener (Lardon, 2003). Le jeu favorise la dynamique de groupes et propose un cadre pour établir, reconnaître ou s'approprier des règles de communication et d'utilisation d'informations. Le jeu contribue aussi à stimuler les raisonnements entre acteurs/joueurs et il facilite l'apprentissage collectif (Angeon et al., 2003). A priori, le raisonnement territorial que permet le jeu de plateau propose donc les mêmes avantages que ceux produits par une cartographie géographique, le plaisir ludique en plus.

\section{Jeux de plateau et problèmes de stratégie}

L'hypothèse de Zhang (2000) sur la complémentarité des représentations externes et internes pour contribuer à la résolution de problèmes a été validée dans le contexte du jeu. Cet auteur a étudié cette hypothèse en observant et en testant, notamment, des parties de Morpion et d'Echecs. Dans une perspective complémentaire, Saucin (2004) a réalisé l'analogie entre le plateau de Go et un territoire de marché en comparant l'objectif territorial du Go "au niveau 
sectoriel à la recherche d'influence sur les produits ou les services (part de marché au niveau des produits ou des services) » (Saucin, 2004). A partir de ces faits, nous proposons une revue de quelques jeux de stratégie sur plateau. Ces jeux ont pour propriétés : d'être assez connus et répandus dans le monde, d'utiliser les deux dimensions du plan, de permettre la mise en œuvre d'une stratégie et d'être relativement complémentaires dans leur mode de représentation des informations. Sous cet angle d'approche, nous présentons d'abord les cinq jeux de plateau suivant : les Échecs, le Go, Othello, le Stratego et le Risk.

\section{Aspects stratégiques et potentiel analogique des Échecs}

Une partie de jeu d'Échecs, comme chacun le sait, se déroule sur un plateau quadrillé en damier de soixante-quatre cases (huit colonnes, huit lignes). La partie emploie six catégories de pièces différentes (Roi, Reine, Tour, Cavalier, Fou et Pion) distinctes par leur couleur pour reconnaître les pièces des deux camps et par les déplacements et les prises (pour le Pion) qu'elles peuvent réaliser. Chaque camp dispose en début de partie de seize pièces (blanches pour l'un et noires pour l'autre) dont l'emplacement de départ est figé dans les règles. Il existe quatre manières de mouvoir les pièces sur l'échiquier : via les lignes et colonnes, via les diagonales, en décrivant un « $\mathrm{L}$ » formé de quatre cases, en roquant (c'est à dire en réalisant un pivot sur la première ligne entre le Roi et une Tour). Pour gagner la partie, il faut capturer le Roi adverse en le mettant sous la menace d'une pièce (c'est-à-dire en échec) et en l'empêchant de se soustraire à cet échec ou à d'autres qui correspondent à l'ensemble de ses capacités de mouvements.

Les principes stratégiques découlant des Échecs sont les suivants (Thiriez, 1995) : 1) Contrôler autant de cases que possible avec chaque pièce, 2) Coordonner au mieux les mouvements des pièces, 3) Protéger chaque pièce menacée avec un nombre de pièces suffisant, 4) Donner plusieurs objectifs à certaines pièces, 5) Immobiliser l'adversaire, 6) Faire jouer l'effet de surprise.

Le jeu d'Échecs permet de mettre en œuvre des stratégies qui peuvent être analogues à celles d'un chef d'entreprise. Il suffit, par exemple, de remplacer selon les contextes, les termes « cases » et « pièce » ci-dessus par ceux de «produit », « équipe », « département », « secteur » ou «marché ». Au niveau des règles, l'analogie peut s'effectuer dans le cas où l'on considère un nombre relativement réduit de pièces différentes par leur capacité de mouvement et où la victoire peut être très rapide et décisive suite à une attaque «surprise ». Concernant le plateau de jeu, ce dernier, peut être assimilé comme bien d'autres à une matrice et, le nombre de cases qu'il contient ainsi que le nombre de pièces en jeu nous laisse supposer qu'il permet de représenter des informations dans un cadre tactique ou stratégique relativement circonscrit.

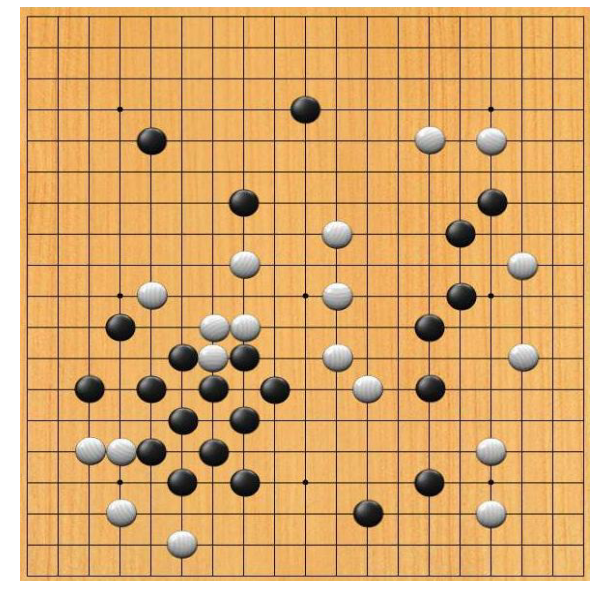

Figure 1. Exemple de partie de Go en cours 


\section{Aspects stratégiques et potentiel analogique au Go}

Une partie de Go se joue sur un «tablier » ou goban habituellement en bois et représentant un quadrillage de dix-neuf lignes horizontales pour dix-neuf verticales (figure 1). Au Go on place les pièces appelées «pierres » sur les trois cent soixante et une intersections du quadrillage. Toutes les pièces sont identiques, mais chaque camp possède des pierres d'une couleur différente de celle de l'adversaire (blanches contre noires). Le déroulement de la partie est le suivant : à chaque tour de jeu, chaque joueur peut poser une de ses cent quatre-vingts pierres sur l'une des intersections qui n'est pas déjà recouverte par une pierre. L'objectif de chaque joueur est de contrôler un maximum d'intersections du goban. Il s'agit en fait de former des «territoires » qui représentent un ensemble d'intersections vides. Pour contrôler un territoire il est nécessaire d'en réaliser le contour. Il est ainsi possible de se servir des limites externes du goban pour fermer un territoire. On peut donc encercler les pièces de son adversaire et ainsi les rendre inutiles. La partie se termine lorsque tous les territoires sont formés et que les frontières sont imprenables. C'est-à-dire qu'il n'y a plus de coup intéressant à jouer. Le joueur contrôlant le plus d'intersections est le vainqueur.

Les principes stratégiques découlant du jeu de Go sont les suivants (Thiriez, 1995) : 1) Chercher une victoire relative plutôt qu'un écrasement de l'adversaire, 2) Savoir prendre appui sur les limites du territoire, 3) Garder à tout moment une vue d'ensemble du jeu, 4) Diviser pour régner, 5) Garder l'initiative, (6) Savoir reconnaître des points vitaux, (7) Disposer de bases fortes, (8) Tenter de jouer des coups à la fois défensifs et offensifs.

Si comme pour les Échecs, nous analysons les analogies qui peuvent être réalisées simplement à partir du jeu de Go, nous pouvons déjà considérer les règles en relations avec les pièces. Le Go ne distingue pas les pièces mises en jeu et ne permet pas de mouvement. Cependant, il permet de prendre en compte un nombre élevé de pions. Au Go la victoire ne peut être complète, puisque, quoiqu'il arrive, l'adversaire contrôlera quelques «territoires » à la fin de la partie. Par contre il est question de stratégie d'enveloppement et de contrôles des extrémités du jeu de préférence à partir du centre. Concernant le plateau de jeu, on joue sur les intersections qui sont très nombreuses par rapport aux Échecs «classiques ». Le Go permet une vision d'une situation a priori à un niveau stratégique plus élevé que celle fournie par les échecs. Du point de vue des stratégies mises en œuvre, le parallèle est encore plus facile à réaliser qu'avec celui des Échecs. Pour se faire, on peut notamment remplacer dans l'énoncé de ses principes stratégiques les termes « jeu » et «territoire » par ceux de «marché » ou de «secteur d'activité ».

\section{Aspects stratégiques et potentiel analogique à Othello}

Le jeu Othello date des années 1970 ; il complète bien sûr le plan stratégique et celui de la représentation des informations les deux jeux précédents. Il utilise un plateau unicolore de soixante-quatre cases, appelé othellier, identique, à la couleur prêt, à l'échiquier classique (figure 2). Comme au Go, chaque joueur pose à tour de rôle un pion en mettant en évidence la couleur qui est la sienne (blanche ou noire). Les pions à Othello sont tous identiques, ils ont deux faces, une blanche et une noire. Le jeu débute au centre de l'othellier avec quatre pions posés sur le carré central en alternant les faces blanches et noires des pions. A chaque tour, un joueur pose un pion sur une case vide (et non une intersection comme au Go) et adjacente à un pion adverse.

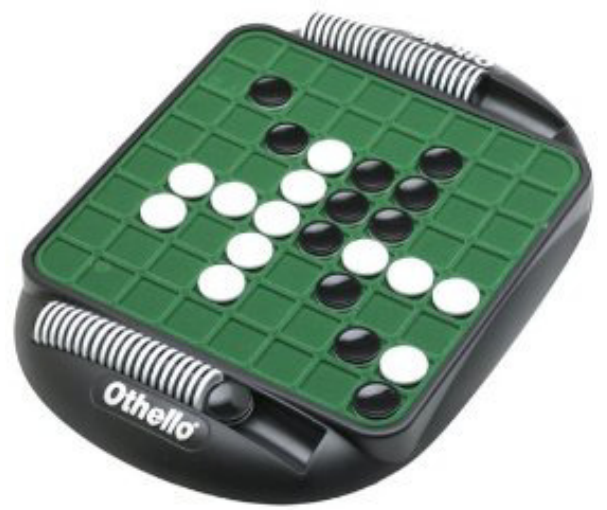




\section{Figure 2. Partie d'Othello en cours}

Il doit également, par cette pose, encadrer avec deux de ses pions au moins un pion adverse. Le pion adverse encadré est alors retourné et change donc de camp. Lorsque l'on ne peut plus jouer de coup, le gagnant est celui qui a le nombre le plus élevé de pions de sa couleur posés sur le plateau.

Les principes stratégiques à Othello sont (Lazard, 2008) : 1) Maximiser le nombre de pions en votre possession non attaquables par l'adversaire, 2) Se préoccuper en début de jeu des zones qui une fois obtenues ne peuvent plus être reprises (les coins à Othello), 3) Garder de vue que certaines zones peuvent changer de propriétaire en cours de partie, 4) Augmentez votre liberté d'action en limitant celle de l'adversaire, 5) Savoir utiliser les frontières pour déployer son jeu à partir d'une position centrale, 6) Renforcer et consolider votre position en jouant sur les bords plutôt qu'au centre (mais, contre un très bon joueur, cette stratégie n'est pas toujours payante), 7) Faire attention aux pièges qui peuvent renverser complètement une situation.

Pour Othello, l'analogie avec le décideur peut être réalisée de la même manière que dans les deux cas précédents. La taille réduite du plateau implique des raisonnements sur certains éléments bien ciblés. A Othello tous les pions sont identiques et ont deux faces. Ils peuvent ainsi rendre compte par analogie, par exemple, de deux choix possibles pour des individus d'un même ensemble. Les règles ne permettent pas réellement de créer des «territoires », mais peuvent rendre compte de renversement de situation, de basculement d'opinion face à une pression de certains pions sur des pions adverses encadrés. Concernant ses principes stratégiques l'analogie avec ceux d'un chef d'entreprise, par exemple, peut être réalisée si l'on utilise les termes « sphère d'influence », « appui », «partenaires » ou «marché » à la place des termes «pion», « zone » ou « jeu ».

\section{Aspects stratégiques et potentiel analogique au Stratego}

Le jeu Stratego date des années 1940, son plateau présente un quadrillage de dix lignes pour dix colonnes moins deux groupes de quatre cases à mi hauteur qui ne forment que deux grandes cases qui font figure de marais infranchissables par toutes les pièces du jeu. Chaque joueur dispose de quarante pions (rouges ou bleus) qui se distinguent par leur force et leur capacité de mouvement ou une capacité spéciale. Les pièces possèdent une face cachée qui empêche un joueur de connaître quel pion adverse occupe telle place (figure 3). Chaque joueur dispose d'un pion Drapeau qui ne peut se déplacer et qui représente le but du jeu. Celui qui prend le Drapeau de l'adversaire gagne la partie. Les Bombes sont les autres pions en possession des joueurs qui ne peuvent être bougés. Les autres pions représentent différents grades militaires de l'Eclaireur au Maréchal d'Empire. Ils forment une échelle de force qui règlemente la victoire d'une pièce sur une autre en cas d'affrontement : le Maréchal d'Empire bat le Général qui bat le Colonel qui bat (...) le Démineur qui bat l'Eclaireur qui bat l'Espion qui ne bat que le Maréchal. Les pions se déplacent selon les lignes et les colonnes du plateau. Chaque joueur joue un pion à son tour et peut choisir d'occuper une pièce où un pion adverse est déjà présent et, de fait l'attaque. Pour attaquer, le joueur doit annoncer le grade de son pion ; s'il est supérieur à celui du pion adverse, il gagne l'affrontement (sauf s'il s'agit d'une bombe et qu'il n'a pas employé un Démineur pour cet affrontement).

D'après la Fédération Internationale de Stratego $^{4}$, ses principes stratégiques sont les suivants: 1) Induire son adversaire en erreur, 2) Rendre ses pièces fortes disponibles pour l'attaque, 3) Identifier les mouvements de l'ennemi pour comprendre la distribution de ses forces, 4) Garder l'initiative, 5) Harceler son adversaire.

Concernant l'analogie du Stratego avec l'environnement du décideur, celle-ci est très facile à imaginer, il suffit de transposer, tels que, les principes du jeu ci-dessus vers l'environnement décisionnel. Concernant le plateau et ses pièces, cet ensemble permet de rendre compte du

4. http://www.isfstratego.com/ 
mouvement d'une variété de pièces un peu plus importante qu'aux Échecs, de jouer sur des variations de poids, de force d'influence, de considérer que certaines cases ne sont accessibles par aucun des camps en présence et de se représenter explicitement l'ensemble de ses pièces en supposant que l'adversaire dispose d'une force équivalente sans être réellement sûr des positions qu'il a choisies. En outre, la position de l'objectif du joueur de Stratego lui est inconnue et protégée par des pions adverses. Ce jeu peut donc servir pour des analogies où l'objectif à atteindre est assez flou et que les choix adverses le sont en partie aussi.

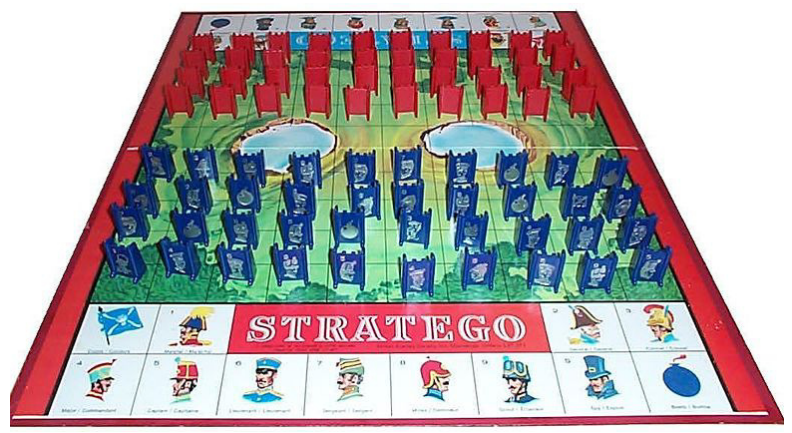

Figure 3. Plateau de Stratego en début de partie

\section{Aspects stratégiques et potentiel analogique au Risk}

Le Risk est un jeu qui date des années 1950 et qui est aussi très répandu dans le monde. Son plateau est constitué d'un planisphère coloré composé de quarante-deux territoires. Les pièces du Risk correspondent à des unités militaires, elles ne se distinguent pas vraiment entre elles, sauf par la couleur désignant le joueur auquel elles appartiennent ainsi que par le nombre d'unités qu'elles représentent. C'est un jeu multijoueur qui peut en intégrer de deux à six, chacun choisissant une couleur spécifique pour ces unités. En début de partie, chaque joueur possède autant d'unités que ses adversaires, mais ce nombre est variable selon le nombre de joueurs (trente unités pour quatre joueurs par exemple). Le jeu utilise aussi deux jeux de cartes. L'un permet de tirer au sort, en début de partie, l'objectif que l'on doit remplir pour gagner la partie, l'autre est utilisé tout le long de la partie pour tirer au sort des ressources militaires à chaque conquête effectuée. Ce dernier jeu de cartes sert aussi à déterminer les territoires que chaque joueur possède en début de partie et sur lesquels il peut disposer ses unités. Chaque joueur dispose alors d'un objectif connu de lui seul qu'il devra remplir le premier pour gagner la partie (la conquête totale de deux ou trois continents, la conquête d'un nombre donné de territoires, l'élimination de l'armée d'un autre joueur, etc.). Au Risk, quand son tour vient, un joueur peut déplacer autant d'unités d'autant de zones contiguës ou reliées par des pointillés qu'il le désire. Pour conquérir un territoire ou zone de terrain du planisphère, il faut d'abord y avoir éliminé les armées adverses. L'assaillant doit annoncer quelle zone il attaque et à partir de quel lieu ; il a alors le choix d'engager jusqu'à trois unités simultanément qui sont représentées chacune par un dé classique à six faces (rouge pour l'attaque et bleu pour la défense). Le défenseur peut lui, engager jusqu'à deux dés sur le même principe. Chacun lance les dés et les scores des deux camps sont comparés. Si l'assaillant a obtenu sur un dès un score supérieur au meilleur du défenseur, le défenseur perd une unité, en cas d'égalité ou d'un meilleur score obtenu par au moins l'un des dés du défenseur, c'est l'assaillant qui perd une à deux armées selon le résultat du jet de dés. Le Risk est donc un jeu à objectif variable (on n'a pas forcément à remplir le même objectif que l'adversaire) et un jeu de stratégie où le hasard intervient de manière notable.

Les principes stratégiques découlant du Risk sont les suivants (d'après notamment l'International RISK Championship ${ }^{5}$ ) : 1) Économiser vos forces, 2) Concentrer vos forces dans certaines zones pour mieux submerger l'adversaire, 3) Détourner l'attention de vos adversaires sur des zones mineures, 4) Savoir cacher votre objectif le plus longtemps possible, 5) Traduire les mouvements de vos adversaires en termes d'objectifs, 6) Faire preuve d'opportunisme (c'està-dire savoir opportunément risquer ses unités), 7) Conserver une réserve stratégique pour l'employer au moment crucial.

5. http://www.risktoc.org/ 


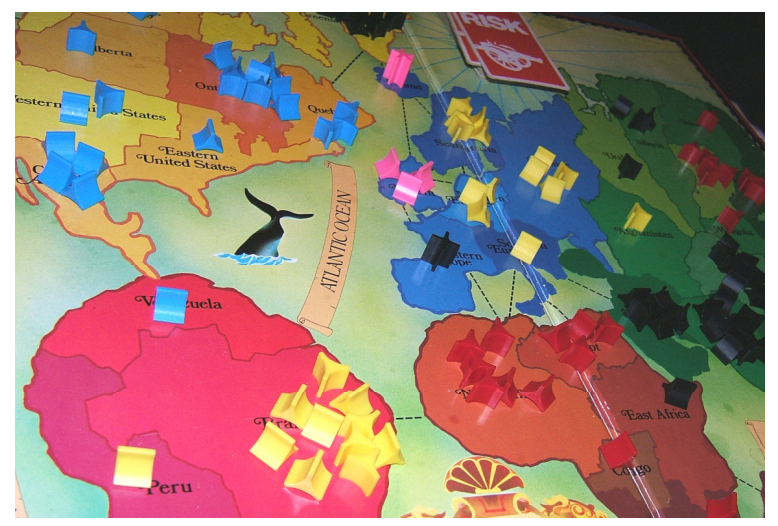

Figure 4. Exemple de partie de Risk en cours

L'analogie entre le décideur et le joueur de Risk est simple à effectuer. Ces principes stratégiques sont ceux de bases de nombreux ouvrages de stratégie militaire (économie et concentration de forces, réserve stratégique, savoir de temps en temps prendre des risques et tenter sa chance, etc.) et plusieurs auteurs ont déjà fait le parallèle entre stratégie militaire et stratégie d'entreprise. Concernant l'analogie au plateau du Risk, ce dernier peut, au minimum, réaliser le parallèle d'un développement en termes de marchés géographiquement déterminés. Nous pouvons aussi illustrer cette analogie possible en reprenant les principes de stratégie du Risk en remplaçant le terme «zone » par ceux de «marché », «segment de marché » ou «secteur d'activité ». Ce jeu permet aussi de représenter la continuité et l'interdépendance de certains segments de marchés et de relativiser les chances de réussites d'un faible déploiement de moyens dans un secteur donné.

\section{Jeux de stratégie sur plateau et wargames}

Le plus ancien jeu de plateau serait le Mehen, il remonterait à la période de l'Égypte ancienne vers l'époque dite Thinite (3100-2700 avant J.C.) (Rothöter, 1999). Il semble que ce soit un jeu appartenant à la même catégorie, par sa forme, que le jeu de l'oie. Depuis cette époque, le jeu de plateau n'a cessé d'évoluer et de proposer diverses variantes dont celle des jeux de stratégie qui nous intéresse ici. Le jeu de plateau a ainsi continué à fournir un moyen de distraction et de réflexion, mais aussi dans l'une de ses évolutions militaire à fournir un outil d'aide à l'enseignement des principes de stratégies militaires ou bien à la simulation d'affrontements pour la prospective ou l'histoire militaire. Sous ce dernier aspect il a donné naissance au « jeu de guerre » ou wargame.

\section{Qu'est-ce qu'un wargame?}

Un wargame est une simulation du déroulement de certaines actions sélectionnées par rapport à une situation de conflits. Il s'agit d'une tentative pour recréer un affrontement réel ou imaginaire dans un espace et un temps définis (Foley et al., 2007). Sa conception est fondée sur des procédures, des règles et des données prédéterminées. Son objectif est d'aider certaines personnes à prendre des décisions, à tester des plans d'opération ou à rechercher, générer et tester de nouvelles idées. Concernant ses origines, celles-ci remontent aux croisements de deux simulations d'affrontements à partir du jeu. La première est issue d'une évolution prussienne du jeu d'Échecs en un jeu plus en rapport avec les réalités d'une bataille qui prit pour nom Échecs de guerre en 1644. La seconde dérive de la représentation d'affrontements réels à l'aide de figurines dont la reconnaissance date de 1773 en Angleterre (Liardet, 1997). À partir de ces deux branches, le wargame sera créé en Prusse sous le nom de kriegspiel entre 1811 et 1824 . Le wargame sur carte topographique est alors mis en œuvre pour former les officiers prussiens. Par la suite, les succès de l'armée prussienne dans la deuxième partie du XIX ${ }^{\mathrm{e}}$ siècle face aux armées 
autrichiennes et françaises populariseront en dehors de l'Allemagne l'emploi du wargame. Peu de temps après les wargames civils commenceront à être développés et complèteront le panel des jeux de stratégie sur plateau. Toutefois, il faudra attendre 1958 pour que la première société dédiée à la création de wargames commerciaux (Avalon Hill) soit créée aux États-Unis.

\section{Caractéristiques des wargames et autres jeux de stratégie sur plateau}

Si l'on souhaite présenter les jeux de stratégie sur plateau, nous pouvons les classer en trois catégories liées au découpage du plateau sur lequel les pions vont être placés. Il existe essentiellement trois formes de découpage d'une carte de jeu de guerre pour y placer des pions : les cartes géomorphiques ou un pavage du plateau est réalisé. Dans cette catégorie, on retrouve essentiellement deux types de découpages: en carrés (Échecs, Go, Othello, Stratego) et en hexagones avec des jeux des comme Les Colons de Catane ou de nombreux wargames commerciaux tactiques comme la série des Great Battles of History ${ }^{6}$ avec des jeux comme Great Battles of Alexander (figure 5). Un autre type de découpage propose des cartes à zones de jeu représentant un découpage le plus souvent en zones géographiques (Age of Napoleon, Diplomacy, Risk...). Les cartes à points de jeu (figure 6) qui utilisent le plus souvent des intersections de voies de communication pour placer les pions (Paths of Glory, Soldier Kings, Successors...). Nous pouvons aussi différencier les jeux de stratégie sur plateau selon les types de placements de pions qu'ils proposent. Il en existe essentiellement deux. Premièrement ces jeux peuvent employer ce que nous nommons des cases qui peuvent être n'importe quelle surface délimitée par des frontières pour la distinguer d'autres surfaces. Les jeux qui utilisent des cases pour placer leurs pièces, sont les Échecs, Othello, le Stratego, des wargames à hexagones comme dans la série Great Battles of History et, des jeux utilisant des zones géographiques comme le Risk.

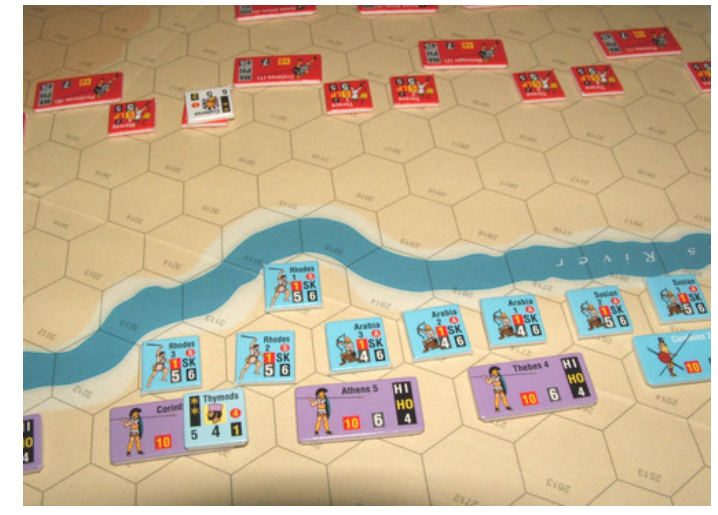

Figure 5. Exemple de reconstitution de la bataille d'Issus à Great Battle of Alexander

Dans la deuxième catégorie, nous retrouvons des jeux où ce sont les intersections de lignes qui servent de repères pour placer les pièces comme dans le cas du Go ou du Xiang $Q i^{7}$ (les Échecs chinois), ou encore des wargames comme Paths of Glory, même si une figure géométrique (carré ou étoile) précise le point d'intersection.

6. Edité par GMT Games.

7. Pour plus d'informations sur ce jeu, voir par ex. : http://www.chine-informations. com/guide/le-xiangqiechecs-chinois_1264.html 


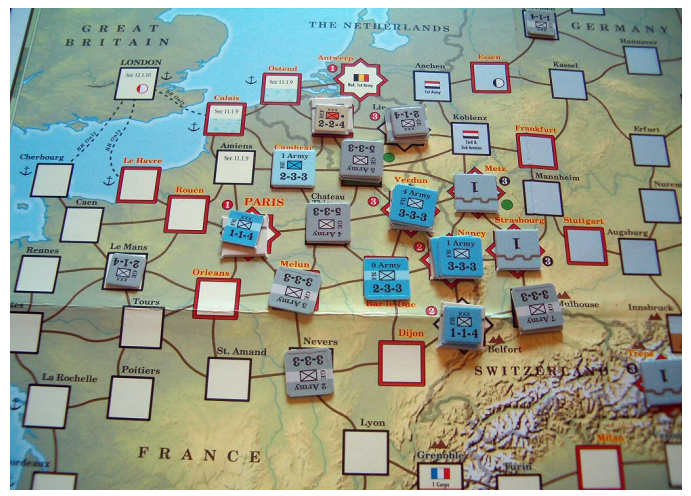

Figure 6. Exemple de carte à points de jeu (Paths of Glory ${ }^{8}$ )

Nous pouvons aussi distinguer les jeux selon que leurs pièces soient visibles par un seul joueur (Stratego, Age of Napoleon) ou tous les joueurs (Échecs, Go, Risk, etc.). Le nombre d'informations distinctes (les valeurs lisibles sur chaque pièce) affichées sur les pions peut aussi permettre de distinguer les différents jeux qui existent. Ainsi, nous avons des jeux où il n'y a qu'une information sur la pièce qui permet de reconnaître son camp d'appartenance (un pion noir au Go, la face blanche d'un pion à Othello). Ils peuvent aussi présenter deux informations représentées le plus souvent par la couleur et la forme de la pièce (au Risk la forme de la pièce indique la taille de l'unité qu'elle représente, tandis qu'aux Échecs elle indique sa capacité de mouvement). Depuis que les wargames commerciaux se sont développés, l'utilisation de pions cartonnés (counters en anglais) s'est largement répandue. Ce sont généralement des pièces carrées de 1,25 cm de côté (ou quelquefois pour des unités moins mobiles, des rectangles de 1,25 $\mathrm{cm}$ de large pour 2,50 $\mathrm{cm}$ de long) représentant une unité militaire (Great Battles of History ou Paths of Glory) et ses caractéristiques (type d'unité, taille de l'unité, capacité de mouvement, valeur en attaque, valeur en défense, capacité spéciale, etc.).

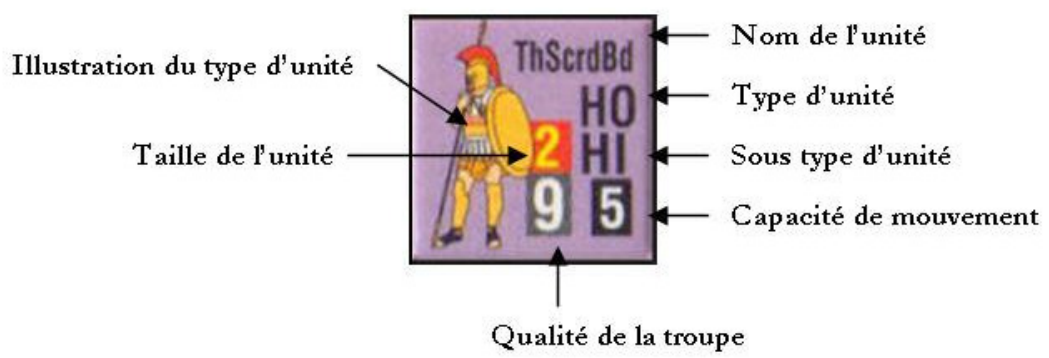

Figure 7. Illustration d'un counter de Great Battles of Alexander

Des pions de forme légèrement différentes existent aussi et sur le même principe ils présentent un peu moins d'informations, comme dans le jeu Age of Napoleon où ils présentent : le nom du général duquel dépend l'unité, une capacité de commandement (combien d'autres pions il est possible de bouger avec ce pion), un potentiel de combat et un potentiel de mouvement). Les jeux présentant trois informations sur leurs pièces sont plus rares ou bien utilisent le même système que les counters des wargames. Nous ne nous attarderons pas sur ces jeux où les pièces ne présentent que trois informations, car ils ne sont pas très courants. Nous citerons à titre informatif le Shogi (les Échecs japonais) qui doit être l'exemple le plus connu et dont les pièces présentent comme informations: une orientation qui caractérise leur camp d'appartenance, un grade sur une face qui renseigne sur les déplacements que la pièce peut 
effectuer et une promotion éventuelle (qui étend les possibilités de déplacements de la pièce) sur l'autre face ${ }^{9}$.

On peut encore distinguer les différents jeux de stratégie sur plateau qui existent par le type de victoire qui est normalement visée par chaque camp (à Othello la victoire totale est possible, mais très rare, donc au départ on recherche une victoire partielle). Le nombre de camps en présence permet aussi de classer les différents jeux. Le nombre d'emplacements possibles sur le plateau peut aussi permettre un classement des différents jeux. Enfin, la nature du fond figurant le plateau permet aussi de les distinguer.

Il peut être «abstrait» comme aux Échecs et au Go ou cartographique, c'est-à-dire représentant une réalité géographique comme au Risk (le planisphère) ou à Great Battles of Alexander (une carte représente les caractéristiques d'un terrain réel).

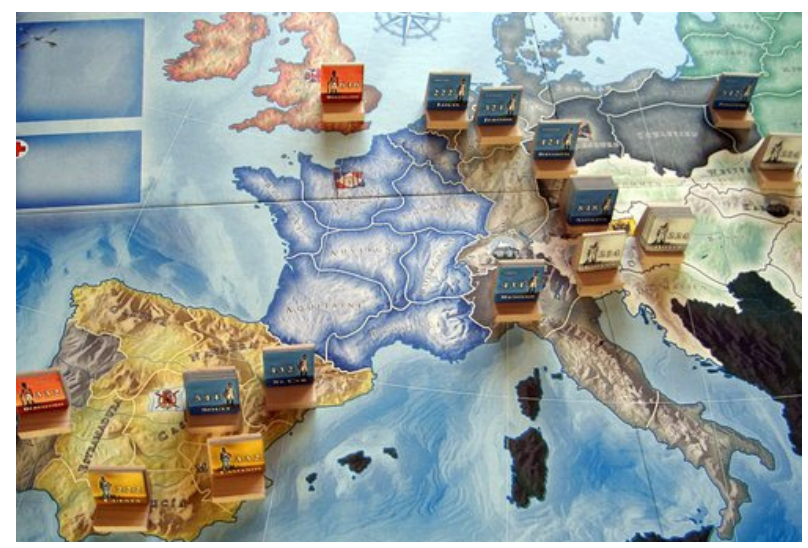

Figure 8. Exemple de carte à zones de jeu (Age of Napoleon) ${ }^{10}$

\section{Conclusion et perspectives pour l'intelligence économique}

Selon Thiriez (1995), il semble exister un lien significatif entre les jeux auxquels les décideurs jouent et leurs décisions stratégiques. Sur cette base, nous pouvons proposer aux décideurs, selon leurs préférences, mais aussi en fonction du problème informationnel considéré, une représentation graphique qui aide à l'explicitation du problème, à la présentation d'une solution ou à la présentation d'une collecte d'informations pertinentes. Selon notre hypothèse de travail et le panel de plateaux de jeux de stratégie que nous avons présentés ici, le spécialiste de l'intelligence économique peut choisir une présentation territoriale d'informations adaptées à un problème informationnel spécifique. Pour se faire, il doit prendre en compte les caractéristiques de l'environnement décisionnel et du décideur. En ce sens, cette proposition contribue aux modélisations actuelles du décideur et de son environnement dans le contexte de l'élaboration d'un système d'information stratégique (Peguiron, 2008). Comme, nous l'avons fait remarquer précédemment, toute représentation n'est pas bonne a priori puisque, soit elle est associée à des représentations internes du lecteur de la carte, soit elle nécessite un apprentissage approfondi des représentations qu'elle fournit pour bien les comprendre et les utiliser. De fait, le spécialiste de l'intelligence économique, avant d'élaborer une carte, se doit de disposer de réponses aux questions suivantes :

- Le décideur accepte-t-il l'idée de l'analogie du jeu de plateau pour enrichir une présentation d'informations?

- Doit-on faire appel à des représentations internes familières au décideur (géographie, plateau de jeu, règles de jeu) pour présenter les informations?

- Quels sont les jeux de stratégie sur plateau que connaît ou apprécie le décideur ?

- Quelle analogie de jeu de stratégie permettra de mieux stimuler les réflexions du décideur?

À partir des réponses à ces questions, si le décideur n'est pas contre l'idée de l'analogie du plateau de jeu de stratégie alors, selon les informations à prendre en compte, le spécialiste de

9. Pour plus d'informations sur ce jeu, voir par exemple le site de l'Association de Shogi d'Alsace: http://shogi.alsace.free.fr/regles.htm

10. Chez Phalanx Games. 
l'intelligence économique doit encore effectuer des choix. En effet, il lui reste à définir, en dehors du contexte décisionnel, quel est la catégorie de représentations cartographiques du type plateau de jeu de stratégie la plus adaptée aux informations qu'il souhaite expliciter ou mettre en valeur. Pour le guider dans cette réflexion, nous avons élaboré un tableau à partir des jeux dont nous avons présenté ci-dessus les caractéristiques et illustrations. Ce tableau reprend l'ensemble des moyens de distinctions des différents jeux que nous avons présenté dans cet article. Nous y avons ajouté deux jeux qui en fait n'existent pas : les Échecs ${ }^{2}$ et le $G o^{2}$. Il s'agit simplement d'un jeu d'Échecs et d'un jeu de Go joués sur un support dont le nombre de lignes et de colonnes a été doublé.

\begin{tabular}{|c|c|c|c|c|c|c|c|c|c|c|c|}
\hline & JEUX & 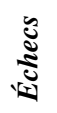 & 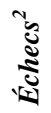 & 8 & కి & 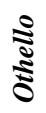 & \begin{tabular}{l}
8 \\
\multirow{3}{*}{} \\
5
\end{tabular} & : & ְ̊ & তี & z \\
\hline \multicolumn{12}{|l|}{ CRITERES } \\
\hline \multirow{4}{*}{ Découpage } & Car & $\mathbf{X}$ & $\mathbf{X}$ & $\mathbf{X}$ & $\mathbf{X}$ & $\mathbf{X}$ & $\mathbf{X}$ & & & & \\
\hline & Hex & & & & & & & & & $\mathbf{X}$ & \\
\hline & Zones & & & & & & & $\mathbf{X}$ & & & $\mathbf{X}$ \\
\hline & $P d J$ & & & & & & & & $\mathbf{X}$ & & \\
\hline \multirow{2}{*}{ Placement } & Case & $\mathbf{X}$ & $\mathbf{X}$ & & & $\mathbf{X}$ & $\mathbf{X}$ & $\mathbf{x}$ & & $\mathbf{X}$ & $\mathbf{X}$ \\
\hline & Inter & & & $\mathbf{X}$ & $\mathbf{X}$ & & & & $\mathbf{X}$ & & \\
\hline \multirow{5}{*}{$\begin{array}{l}\text { Nombre de } \\
\text { places } \\
\text { disponibles }\end{array}$} & $60<$ & & & & & & & $\mathbf{X}$ & & & $\mathbf{X}$ \\
\hline & $120<$ & $\mathbf{X}$ & & & & $\mathbf{X}$ & $\mathbf{X}$ & & & & \\
\hline & $300<$ & & $\mathbf{X}$ & & & & & & $\mathbf{X}$ & & \\
\hline & $700<$ & & & $\mathbf{X}$ & & & & & & & \\
\hline & $>700$ & & & & $\mathbf{X}$ & & & & & $\mathbf{X}$ & \\
\hline \multirow{2}{*}{$\begin{array}{l}\text { Valeurs } \\
\text { des pièces } \\
\text { visibles par }\end{array}$} & Tous & $\mathbf{X}$ & $\mathbf{X}$ & $\mathbf{X}$ & $\mathbf{X}$ & $\mathbf{X}$ & & $\bar{X}$ & $\mathbf{X}$ & $\mathbf{X}$ & \\
\hline & Un seul & & & & & & $\mathbf{X}$ & & & & $\mathbf{X}$ \\
\hline \multirow{3}{*}{$\begin{array}{l}\text { Nombre } \\
\text { d'informa- } \\
\text { tions porté } \\
\text { par pièce }\end{array}$} & 1 & & & $\mathbf{X}$ & $\mathbf{X}$ & $\mathbf{X}$ & & & & & \\
\hline & 2 ou 3 & $\mathbf{X}$ & $\mathbf{X}$ & & & & $\mathbf{X}$ & $\mathbf{X}$ & & & \\
\hline & 4 et + & & & & & & & & $\mathbf{X}$ & $\mathbf{X}$ & $\mathbf{X}$ \\
\hline \multirow{2}{*}{$\begin{array}{l}\text { Nombre de } \\
\text { camps }\end{array}$} & 2 & $\mathbf{x}$ & $\mathbf{x}$ & $\mathbf{x}$ & $\mathbf{X}$ & $\mathbf{X}$ & $\mathbf{X}$ & & $\mathbf{X}$ & $\mathbf{x}$ & $\mathbf{x}$ \\
\hline & 3 et + & & & & & & & $\mathbf{x}$ & & & \\
\hline \multirow{2}{*}{$\begin{array}{l}\text { Victoire } \\
\text { visée }\end{array}$} & Partielle & & & $\mathbf{X}$ & $\mathbf{X}$ & $\mathbf{X}$ & & & $\mathbf{X}$ & $\mathbf{X}$ & $\mathbf{X}$ \\
\hline & Totale & $\mathbf{X}$ & $\mathbf{X}$ & & & & $\mathbf{X}$ & $\mathbf{X}$ & & & \\
\hline \multirow{2}{*}{ Fond } & Abstrait & $\mathbf{x}$ & $\mathbf{x}$ & $\mathbf{x}$ & $\mathbf{X}$ & $\mathbf{X}$ & $\mathbf{X}$ & & & & \\
\hline & Carte & & & & & & & $\bar{x}$ & $\mathbf{X}$ & $\mathbf{X}$ & $\mathbf{x}$ \\
\hline
\end{tabular}

Tableau 1. Récapitulatif des éléments constitutifs des différents jeux présentés ${ }^{11}$

À travers l'intégration de ces jeux «virtuels » dans ce tableau, notre objectif est de fournir une aide pour envisager l'agrandissement d'un quadrillage de type Échecs ou Go et de se rendre

11. Légende $:$ Car $=$ carrés, Hex $=$ hexagones, $\mathrm{PdJ}=$ points de jeu, Inter $=$ intersection, PoG $=$ Paths of Glory, GBH = Great Battles of History, AoN = Age of Napoleon. 
compte du nombre d'emplacements qu'ils permettraient d'utiliser comparativement à d'autres plateaux. Ceci dit, l'équivalent des Échecs ${ }^{2}$ existe bien puisque les Échecs japonais ont été déclinés sous la forme d'un grand Shogi (Dai Shogi) dont le nombre de cases est de quinze par quinze, et qu'en outre, d'autres Shogi encore plus grands (jusque vingt-cinq lignes pour vingtcinq colonnes) ont aussi été élaborés (Reysset et al., 2000).

Au final, nous pensons qu'à partir de ce genre de représentations graphiques, un spécialiste de l'intelligence économique dispose d'une opportunité supplémentaire pour ajouter de la valeur aux informations collectées à travers leur présentation sous la forme de cartes réalisées par analogie aux jeux de plateau. Dans cette optique, nous compléterons ce tableau pour le rendre plus complet et mieux catégoriser les jeux sur plateau. Désormais, nous travaillons à l'élaboration d'un logiciel intégrant l'ensemble des paramètres présentés ci-dessus. Nous comptons aussi tester l'emploi de ces cartes selon qu'on les affiche via un écran d'ordinateur, une table tactile ou en les imprimant sur des pages au format A2 ou A3. Nous souhaitons aussi affiner par le biais de cette expérimentation les critères de choix des cartes ainsi que préciser les modalités de l'analogie à mettre en place selon le jeu d'origine. À moyen terme, notre expérimentation pourra aussi nous amener à étudier la transition d'une simple représentation graphique vers un outil d'apprentissage, voir de simulation comme c'est le cas pour les wargames.

\section{Bibliographie}

AFDIE, Les cahiers de l'AFDIE (Association française pour le développement de l'intelligence économique), $\mathrm{n}^{\circ}$ 7, Paris, 2005.

Angeon V., Lardon S., «Dessiner et comprendre le territoire : quand le jeu devient un processus collectif d'apprentissage et de création », Les figures du projet territorial, sous la direction de B. Debardieux et S. Lardon, 2003, p. 245-257.

Anthony S.D., Eyring M., Gibson L., "Mapping your Innovation Strategy", Harvard Business Review, May, 2006, p. 1-10.

Arndt, J., "On Marketing Science More Scientific: Role of Orientations, Paradigms, Metaphors, and Puzzle Solving", Journal of Marketing, n²9, 1985, p. 11-23.

Bizet F., Bussi M., «Les jeux de plateau : une géographie ludique », Mappemonde, 4, 1997, p. 33-37.

Borzakian M., «Pour une géographie des jeux de plateau », Cybergeo : European journal of Geography, 2009.

Bouzdine-Chameeva T., Ferrand A., «Un produit nouveau pour une marque existante : l'analyse d'interaction des champs sémantiques d'une perspective cognitiviste à travers la cartographie causale », Actes des $3^{e}$ ateliers de la Recherche en Design, Bordeaux, 2007.

Buisine S., Eveque L., Fouladi K., Marlier J., Turner W.A., «Représentation d'un espace de valeurs d'usage pour guider la conception », Actes du colloque Ingénierie de la Connaissance, Hammamet, 2009.

Casakin H., Goldschmidt G., "Expertise and the use of visual analogy: implications for design education", Design Studies, vol. 20, issue 2, March 1999, p. 153-175.

Clerc P., «Les dispositifs territoriaux d'intelligence économique: Analyse comparée du Japon, du Royaume Uni, du Canada et de la France », Regard sur l'IE, n 1, février 2004.

David A., Thiery O., «L'architecture EQUA2TE et son application à l'intelligence économique », Actes de la conférence IERA (intelligence économique, recherches et applications), Nancy, 2003.

Delvoye J.M., Girardot J.J., «Les outils de l'intelligence territoriale pour les acteurs de terrain à Seraing: entre appropriation des méthodes et acquisition de compétences », Proceedings of the International Conference of Territorial Intelligence in the framework of the CAENTI, Liège, 2005.

Eppler M., Burkhard R., "Using Visual Representations in Knowledge Management: a Conceptual Framework and Application Examples", Proceedings of the MKWI 2006: Minitrack on Visualization Methods for KM Applications, 2006.

Fauvet J.C., Smia M., Le manager joueur de go, Eyrolles, Paris, 1997.

Galland S., Boulanger N., Rostiang H., «L'implication des experts dans un processus de prise décision », Benchmark Européen des pratiques en intelligence économique, 2008, p. 104-115 
Gick M.L., Holyoak K.J., «Schema induction and analogical transfer », Cognitive Psychology, $\mathrm{n}^{\circ} 15$, 1983, p. 1-38.

Goria S., «Entre la veille stratégique et l'innovation, la démarche de veille créative : Ce que la veille créative emprunte aux wargames sur plateau », Actes du séminaire international Veille Stratégique Scientifique et Technologique, VSST'2009, Nancy, 2009.

Goria S., «Expression de problème, Explicitation de connaissances ou de solutions nouvelles, Représentation graphique d'information: un triptyque clé pour l'étudiant en intelligence économique », Actes des assises nationales de la formation en intelligence économique, Aix en Provence, 2008.

Goria S., L'expression de problème et la médiation informationnelle: le cas posé par l'intelligence territoriale, Editions VDM Verlag Dr. Müller, 2007.

Goria S., Knauf A., «Présentation d'une étude fonctionnelle de diverses formes d'Intelligences Territoriales mises en œuvre par les pôles de compétitivité : Vers l'identification de nouvelles prestations », Actes du Colloque international, Pôles de compétitivité et développement économique régional Liège, 2009.

Guilhon A, Le processus d'intelligence économique et l'identité de la PME, L'intelligence économique dans la PME : visions éparses, paradoxes et manifestations, L'harmattan, p. 21-42, 2004.

Humbert P., François C., Cuxac P., David A., «La visualisation des connaissances scientifiques : intégration des besoins des utilisateurs », The Canadian Journal of Information and Library Science (CJILS), vol. 30, $\mathrm{n}^{\circ} 1-2$, septembre 2008, p. 37-50.

Hunt S.D., Menon A., "Metaphors and competitive advantage: evaluating the use of metaphors in competitive strategy", Journal of Business Research, $\mathrm{n}^{\circ} 33,1995$, p. 81-90.

Joachim J., Kister J., Bertacchini Y., Dou H., «Intelligence économique \& système d'information », International Journal of Information Sciences for Decision Making, n 24, 2006.

Foley C., Pierre-Pierre A., «Qu'est-ce que le wargame ? Une introduction rapide », Vae Victis, Revue du Jeu d' Histoire Tactique et Stratégique, HS Nº6, 2007, p.4-11.

Lancini A., Lebraty, J.F., «Contributions des systèmes d'information centrés réseau en situation de crise : Le cas d'une gestion de crise agro-alimentaire différenciée entre Leclerc et Nestlé », Actes du séminaire ENITIAA (École nationale d'ingénieurs des techniques des industries agricoles et alimentaires) 2007: Instabilité des systèmes d'information et de décision dans les organisations, Nantes, 2007.

Lardon S., «Diagnostic de territoire et représentations spatiales : les chorèmes, graphes et jeux », Les figures du projet territorial, sous la direction de B. Debardieux et S. Lardon, DATAR, 2003, p. 109129.

Lardon S., Caron P., Bronner A.C., Giacommel G., Raymond R., Brau F., «Jeu collaboratif de construction de territoire: interagir par les représentations spatiales », Revue internationale de géomatique, vol. 18, $\mathrm{n}^{\circ}$ 4, 2008, p. 507-530.

Lardon S., Maurel P., Piveteau V., Représentation spatiales et développement territorial, Hermès, 2001.

Lazard E., A la découverte d'Othello : Manuel d'initiation, Fédération française d'Othello, 2008.

Lecocq R., «La cartographie des connaissances », Gestion stratégique des connaissances, dir. L. Rivard et M.C. Roy, Presses de L’Université de Laval, 2005, p. 53-64.

Lempert E., Espace et géostratégie, Hermès, 34, 2002.

Lendrevie J., De Baynast A., Publicitor : Théorie et pratique de la communication, Dalloz, 2004.

Liardet J.P., Les wargames commerciaux américains des années soixante à nos jours, entre histoire militaire et simulation, une contribution à l'étude de la décision, Septentrion Presses Universitaires, 1997.

Martre H., Levet J.L., Clerc P., Intelligence économique et stratégie des entreprises, La Documentation française, 1994. 
Nogry S., Didierjean A., «Les erreurs commises lors de la résolution du problème source favorisent-elles le transfert analogique? Un réexamen de la recherche de Gick et McGarry (1992) », Psychologie Française, vol. 52, issue 3, September 2007, p. 341-353.

Pautrat R., Delbecque E., «L’intelligence territoriale : la rencontre synergique public/privé au service du développement économique », Revue Internationale d'intelligence économique, 1, 2009, p. 17-28.

Peguiron F., L'intelligence économique au service des acteurs de l'Université : La question du partage de l'information sur les campus, L'Harmattan.

Reysset P., Cazeaux J.L., L'univers des Échecs, Éditions Bornemann, Paris, 2000.

Romma N., Boutin E., «Les stratégies d'influence sur Internet: validation expérimentale sur le lobby antinucléaire », Actes du Colloque Ile Rousse : Journée sur les systèmes d'information élaborés, 2005.

Rothöter B., Mehen, “God of the Boardgames”, Board Games Studies, vol 2, 1999, p. 10-59.

Saucin J., Le jeu de Go, modèle analogique pour les sciences humaines, Les certitudes de l'Aurore, Bruxelles, 2004.

SCIE (Service de coordination à l'intelligence économique), Guide des bonnes pratiques en matière d'intelligence économique, février 2009.

Slywotzky A., Patterns, 30 dynamiques de profit, Village Mondial, 1999.

Thiriez H., Jeux, Cultures et Stratégie, Les Éditions d'Organisation, 1995.

Vinck V., Ingénieurs au quotidien. Ethnographie de l'activité de conception et d'innovation, Presses Universitaires de Grenoble, Grenoble, 1999.

Zhang J., "External Representations in complex Information Processing Tasks", Encyclopedia of Information Science, vol. 68, New York, Marcel Dekker, Inc., 2000. p. 164-8. 